\title{
PRESENTACIÓN DEL MONOGRÁFICO: VALIDACIÓN DE CONTENIDO DESDE METODOLOGÍAS CUALITATIVAS Y CUANTITATIVAS INTRODUCTION TO THE MONOGRAPH: CONTENT VALIDATION FROM QUALITATIVE AND QUANTITATIVE METHODOLOGIES
}

\author{
Francisco Pablo Holgado Tello y María Isabel Barbero García \\ (Coordinadores) \\ UNED
}

Cómo referenciar este artículo/How to reference this article:

Holgado-Tello, F. J. y Barbero-García, M. I (2013). Presentación del monográfico: Validación de Contenido desde metodologías cualitativas y cuantitativas [Monographic Introduction: Content Validation from Qualitative and Quantitative Methodologies]. Acción Psicológica, 10(2), 1-2. http://dx.doi.org/10.5944/ ap.10.2.11819

La obtención de evidencias de validez de contenido se torna en un aspecto central en la construcción de cualquier instrumento de medida ya sea estandarizado, semi-estandarizado, o no estandarizado. Un buen y correcto estudio de validación de contenido va a tener importantes implicaciones y consecuencias positivas en todas las fases de elaboración del instrumento de medida. Va a permitir desde definir claramente el concepto a medir y operativizarlo en indicadores medibles, hasta facilitar, por ejemplo, que las soluciones factoriales que se encuentren sean coherentes con las hipótesis de partida (en el caso de instrumentos estándares y semi-estándares). Afortunadamente, el abanico de técnicas y procedimientos susceptibles de ser usadas para la obtención de evidencias de validez de contenido es amplísimo, pudiendo usarse desde entrevistas en profundidad, hasta métodos estadísticos que originan escalas de intervalo. Esta circunstancia implica que el uso conjunto de metodologías cualitativas y cuantitativas se complementen para aportar matices que hacen que las evidencias de validez de contenido así obtenidas sean cualitativa y cuantitativamente de me- jor calidad, permitiendo de esta forma una mayor correspondencia teoría-dato.

El objetivo de este monográfico es doble, en primer lugar enfatizar la relevancia que en la construcción de instrumentos de medida necesariamente ha de tener el estudio de la validez de contenido; y en segundo lugar mostrar teórica y empíricamente formas de obtener evidencias de validez de contenido mediante las aportaciones presentadas.

El monográfico consta de nueve contribuciones teóricas, básicas y prácticas. En primer lugar, se realiza una extensa y exhaustiva revisión del devenir histórico del concepto de validez de contenido y de cómo obtener evidencias empíricas sobre ello. Sin duda se trata de un artículo esencial para poder centrar el tema que nos ocupa y obtener una excelente fotografía del momento en el que nos encontramos respecto a la validación de contenido. A continuación, se presenta un estudio básico sobre el método de Osterlind para la obtención de evidencias de validez de contenido. Se trata de uno de los métodos más usados, quizás por la sencillez y los buenos resultados que ofrece, por lo que una revisión del mismo es necesaria, 
concretamente se aportan las principales características diferenciadoras de uso en función del carácter politómico de las respuestas dadas en el Juicio de Expertos. En tercer lugar, demostrando que la validez en general, y en concreto la de contenido, no sólo es importante en Psicología, se presenta un estudio aplicado en el campo de la Responsabilidad Social Corporativa. Concretamente, se analiza la validez de un conjunto de indicadores en sus respectivas dimensiones utilizando el Análisis Jerárquico como técnica estructurada para analizar entrevistas y se pone en relación con métodos de escalamiento como el de las Comparaciones Binarias de Thurstone. En este sentido, se entiende que la validación de contenido supone un ordenamiento escalar de los ítems respecto a su relevancia y representatividad, y en tanto que se obtendría una escala ordenada de ítems, la validación de contenido se vería beneficiada de las distintas propiedades métricas de las escalas de medida, como las de intervalo, por ejemplo. En cuarto lugar, se presenta un estudio de validación de contenido en un área tan compleja como es la violencia de género, donde se aplican distintas técnicas con el objetivo de obtener una escala que evalúe el grado de violencia de género en adolescentes. Como quinto trabajo, encontramos una aplicación del índice de Osterlind para la adaptación de una escala en la evaluación de la envidia en adultos. El sexto trabajo presentado lleva la cuestión más allá de la construcción de instrumentos estandarizados y semi-estandarizados y plantea la validación de contenido en la construcción de instrumentos no-estándares. Mediante la Teoría de la Generalizabilidad se construye y depura un instrumento de observación de funciones docentes. En séptimo lugar, se muestra un muy buen ejemplo de complementariedad entre metodologías cualitativas y cuantitativas. Se presenta el desarrollo de un cuestionario que mide el afrontamiento ante el estigma de personas con VIH, para ello utiliza entrevistas en profundidad para analizar las principales estrategias de afrontamiento usadas, tras lo cual se diseña un cuestionario cuya estructura factorial se coteja para analizar la correspondencia teoría-dato. En penúltimo lugar, y siguiendo desde un enfoque predominantemente cualitativo, se analizan los principales componentes del clima laboral en un contexto sanitario. Para ello, se utilizan entrevistas en profundidad a informantes claves y el método denominado Teoría Fundamentada. Finalmente, el último trabajo trata de hacer evidente que dentro del proceso de adaptación de instrumentos también es necesario obtener evidencias que pongan de manifiesto que los dominios utilizados sean relevantes y estén muestreados de forma representativa. Este aspecto, se concreta en la adaptación a población infantil del Test Neuropsicológico de Aprendizaje y Memoria Visual.

Nos gustaría que el monográfico sirva a su propósito, y que ponga de relieve la importancia de cuidar en extremo la obtención de evidencias de validez de contenido. Sin duda alguna, debería adquirir un papel protagonista en la construcción de cualquier instrumento de medida, ya sea estándar, semi-estándar, o no estándar, y en cualquier área de conocimiento. En este sentido, nos gustaría enfatizar la necesidad de cuidar el diseño y ejecución de los estudios de validación de contenido.

Dedicatoria.-Siempre es difícil escribir una dedicatoria, pero en este caso aún más por la carga emocional que implica dedicar este monográfico a José Antonio Pérez-Gil. Al margen de que el origen de este trabajo fuera iniciativa suya, y que sólo esta circunstancia ya lo merecería, se trata de un amigo del que nos hubiera gustado aprender más y conocer mejor, y a quién siempre hemos admirado por su intuición, inteligencia, y sobre todo por haber sido coherente con su vida. 